\title{
Tuning of spontaneous emission of two-dimensional photonic crystal microcavities by accurate control of slab thickness
}

\author{
A. R. Alija, L. J. Martínez, A. García-Martín, and M. L. Dotor \\ Instituto de Microelectrónica de Madrid, Centro Nacional de Microelectrónica, Consejo Superior de \\ Investigaciones Científicas, Isaac Newton 8, PTM Tres Cantos, 28760 Madrid, Spain \\ D. Golmayo \\ Instituo de Ciencia de Materiales de Madrid-Consejo Superior de Investigaciones Científicas (CSIC), \\ Cantoblanco, 28049 Madrid, Spain \\ P. A Postigo a) \\ Instituto de Microelectrónica de Madrid, Centro Nacional de Microelectrónica, Consejo Superior de \\ Investigaciones Científicas, Isaac Newton 8, PTM Tres Cantos, 28760 Madrid, Spain
}

(Received 8 September 2004; accepted 15 February 2005; published online 29 March 2005)

\begin{abstract}
We have found a blueshift in the cavity modes confined in two-dimensional photonic crystal microcavities when the thickness of the slab was varied uniformly by accurate dry etching. The shifts in the wavelength of the cavity modes were around $2 \mathrm{~nm}$ towards shorter wavelengths per nanometer reduced in the thickness of the slab. Three-dimensional plane wave expansion calculations showed that the observed shifts are inside the calculated photonic band gap of the structures. The variation in the energy position of the peaks with the thickness has been analyzed by three-dimensional finite difference time domain calculations for a one missing hole microcavity. This tuning of the emission wavelength with the change in the thickness slab shows the important effect of the third dimension in photonic crystals made out of semiconductor slabs and it can be of interest for its application in the final processed photonic devices like photonic crystal lasers. (C) 2005 American Institute of Physics. [DOI: 10.1063/1.1896427]
\end{abstract}

Two-dimensional (2D) photonic crystal (PC) slabs have attracted much attention due to the important applications in photonic devices such as $2 \mathrm{D}$ photonic crystal lasers ${ }^{1}$ and photonic crystal waveguides. ${ }^{2}$ The planar technology used for their fabrication contributes to increase the interest in these so called $2 \mathrm{D}$ photonic crystal devices, aiming at the fabrication of high scale integrated optics and optoelectronics. There are numerous experimental studies on the characterization of the spontaneous emission of photonic crystal micro and nanocavities ${ }^{3}$ for their application in different types of photonic crystal lasers ${ }^{4}$ as well as light-emitting diodes. ${ }^{5}$ All of those works focused mainly on the variation of the photonic properties as the shape of the cavities, ${ }^{6}$ the symmetry of the lattice ${ }^{7,8}$ or the main parameters of the periodic lattice ${ }^{8}$ - lattice parameter $a$ and filling factor $f$-were changed.

Nevertheless, there is limited work on the effect of the third dimension (in this case the thickness of the slab) on the photonic band gap properties of a slab. ${ }^{9}$ This important issue has been treated from a theoretical point of view before in waveguides $^{10}$ or pure slabs. ${ }^{11}$

In this work we have studied the variation of the spontaneous emission spectra of photonic crystal microcavities when the thickness of the slab in which they are made is changed. Here we demonstrate that by accurate etching of the PC slab the frequencies of the cavity modes of the PC microcavities can be tuned in the order of tens of nanometers towards higher energies. This finding may have important application in the tuning of PC lasers ${ }^{12}$ as the ones used for chemical detection $^{13}$ without having to change the main

\footnotetext{
a) Author to whom correspondence should be addressed; electronic mail: aitor@imm.cnm.csic.es
}

parameters - lattice parameter $a$ and filling factor $f$-of the fabricated structure.

A 2D PC structure with a triangular lattice of air holes has been fabricated on a slab of InGaAsP pseudo-quaternary semiconductor material. The slab was grown on an InP (001) substrate by solid-source molecular beam epitaxy. The heterostructure consisted of three quantum wells (QWs) of $\mathrm{In}_{0.47} \mathrm{Ga}_{0.53} \mathrm{As}$ of $7.3 \mathrm{~nm}$ separated by 20 -nm-thick barriers and embedded into the waveguide layers. Barriers between QWs and waveguide layers are made of $\left(\mathrm{Ga}_{0.47} \mathrm{In}_{0.53} \mathrm{As}\right)_{4} /(\mathrm{InP})_{5}$ short period superlattices that replace the quaternary alloy enhancing control of the alloy composition and carrier confinement. ${ }^{\mathrm{T}}$

This specific heterostructure gives rise to a strong photoluminescence (PL) spectra centered around $1500 \mathrm{~nm}$ at room temperature which is sufficiently wide as to probe the optical characteristics across a wavelength range of $\sim 300 \mathrm{~nm}$. The original total thickness of the heterostructure was $265 \mathrm{~nm}$. We have chosen a slightly thicker structure than the otherwise standard thickness of $\lambda / 2 n$ in order to cover a range in the variation of the thickness from $\sim 1.17 \lambda / 2 n$ to $\sim 0.95 \lambda / 2 n$ for $\lambda=1.5 \mu \mathrm{m}$ and $n=3.3$ and avoiding damage to the QWs due to the etching.

Processing of the PC structures was done by electronbeam lithography of a poly(methylmethacrylate) layer on top of a $\mathrm{SiO}_{2}$ layer (300 nm thick) deposited by plasmaenhanced chemical vapor deposition. Reactive ion beam etching was used to open the holes in the $\mathrm{SiO}_{2}$ and the semiconductor by $\mathrm{CHF}_{3} / \mathrm{N}_{2}$ and $\mathrm{CH}_{4} / \mathrm{H}_{2}{ }^{15}$ combined with oxygen plasma. Circular holes are then drilled in the InGaAsP membrane to form a 2D triangular PC array in which some holes are omitted. To make the microcavities, $12 \mathrm{PC}$ rows surround hexagonal cavities with a variable number of miss- 


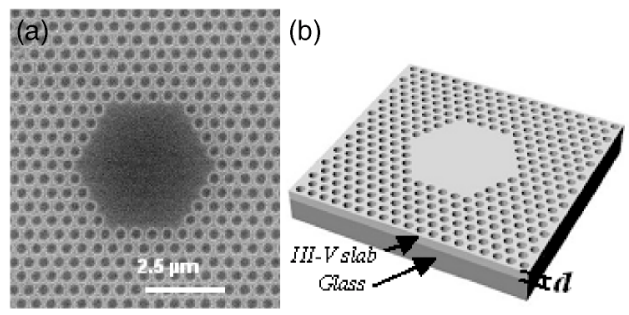

FIG. 1. (a) Scanning electron microscopy image of the fabricated H5 cavities. (b) Schematic view of the cavity fabricated on the III-V semiconductor slab with a thickness $d=265 \mathrm{~nm}$ bonded to a glass substrate.

ing holes per side (one for a $\mathrm{H} 1$ cavity, three for a $\mathrm{H} 3$ and five for a H5 cavity). The period of the lattice $a$ is $500 \mathrm{~nm}$, whereas the filling factor $f$ is around 39\%, changing slightly between different cavities. Fluctuations in the size of different holes due to fabrication were estimated from scanning electron microscopy (SEM) image measurements around 5\% of the $f$ value. In order to provide the membranes with a sufficiently strong mechanical support to resist a number of repetitive steps of dry etching and optical characterization, we bonded the fabricated layers to a thin borosilicate glass $(n=1.53)$ with optical glue $(n=1.5)$ as reported by other authors. ${ }^{16}$ Finally, the InP substrate was etched in a $4: 1$ $\mathrm{HCl}: \mathrm{H}_{2} \mathrm{O}$ solution to leave the semiconductor slab exposed to the air. Figure 1 shows a scanning electron microscopy image of one H5 cavity and a descriptive drawing of the fabricated structure.

The experimental characterization was performed by optical microphotoluminescence spectroscopy. ${ }^{17}$ A $780 \mathrm{~nm}$ laser diode was focused on the sample surface through a 0.4 numerical aperture microscope objective. The size of the excitation spot was around $3.5 \mu \mathrm{m}$ which is small enough to fit inside the PC structures and to generate cavity modes. Light is collected by a lens inside a $0.22 \mathrm{~m}$ monochromator with a cooled InGaAs photodiode connected to a lock-in amplifier. The resolution of the experimental setup in this configuration is around $2.5 \mathrm{~nm}$. Figure 2 shows the evolution of the photoluminescence spectra from the same $\mathrm{H} 5$ cavity after several steps of decreasing the thickness of the slab by $\mathrm{CH}_{4} / \mathrm{H}_{2}$ reactive ion beam etching. The spectra obtained on an unpatterned area in the immediate vicinity of the cavity is also shown. Different emission peaks corresponding to different cavity modes were observed depending on the thickness of the slab. The spectra of the unpatterned area changes their intensity due to the decrease of the total volume, which reduces the number of photogenerated carriers. Comparison between the spectra shows that all of the peaks shift to higher normalized frequencies $(\omega a / 2 \pi c)$ as the slab thickness decreases. All cavities spectra showed a background photoluminescence intensity which is related to three facts: the use of an excitation laser diode in continuous wave modewhich heats up the material considerably although it is more convenient than a pulsed laser to produce higher PL intensity - , the use of a glass substrate-for better mechanical resistance than that of an air suspended membrane-and the detection of the PL intensity that exits from the cavity in normal incidence to the surface, and which is not affected by the band gap confinement. This background photoluminescence intensity decreases as the slab gets thinner. The number of detected cavity modes (whose frequencies are very close in an H5 cavity) are limited by the mentioned backDownloaded 05 Nov 2009 to 161.111.235.169. Redistribution subject

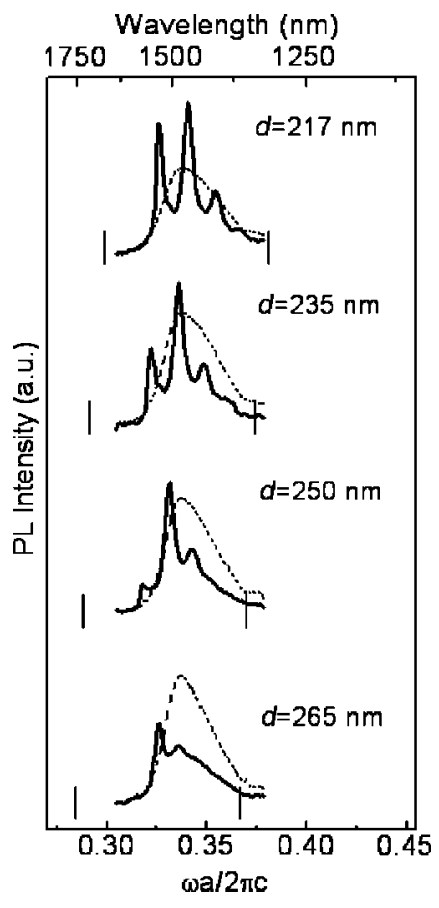

FIG. 2. Photoluminescence spectra of the same H5 microcavity as the thickness of the slab $d$ is decreased (solid line). The spectra of an unpatterned region in the vicinity is shown also (segmented line). Straight lines on each of the spectra mark the position of the photonic band gap calculated by three-dimensional PWE method.

ground PL, the spectral width of the PL spectrum of the material, the resolution of the experimental setup used, and by the quality of the sample. Nevertheless, we detect a sufficient number of modes as to show clearly the relevance of the third dimension in the actual photonic band gap structures. Figure 2 includes the normalized frequencies of the edges of the calculated photonic band gap as the slab gets thinner. These were obtained by a three-dimensional (3D) plane wave expansion (PWE) method for a triangular lattice with the same lattice parameter and filling factor. For this purpose, we used a 3D supercell comprising the semiconductor slab surrounded by $750 \mathrm{~nm}$ of glass on bottom and $750 \mathrm{~nm}$ of air on top. We checked the value of the filling factor by SEM pictures of the initial surface, obtaining a $r / a$ value of $0.328(a=500 \mathrm{~nm})$. To make the calculations as realistic as possible, we have considered that the holes have a slightly tapered shape with tilted walls at an angle of $5^{\circ}$ respect to the vertical direction. This angle is typically obtained in vertical walls etched by $\mathrm{CH}_{4} / \mathrm{H}_{2}$ reactive ion beam etching. ${ }^{18}$ A similar value was obtained in other samples fabricated in our etching system. ${ }^{19}$ The value of this angle is important as it affects losses in photonic crystals waveguides ${ }^{10}$ and $Q$ factor of cavities. In our case the change in the $Q$ factor (which is $<1000$ ) as the thickness changes is not big enough by itself as to produce the observed shift in the modes, as will be explained bellow. Figure 2 shows that the band gap shifts to higher normalized frequencies as the slab gets thinner. The edge of the conduction band shifts at a slightly faster rate than the edge of the valence band (i.e., the band gap gets slightly wider), allowing the cavity modes to appear inside the cavity as the gap shifts.

Figure 3 shows the change in the emission (normalized frequencies) of the observed cavity modes for the same H5 cavity of Fig. 1 versus the etched thickness. The rate of to AIP license or copyright; see http://apl.aip.org/apl/copyright.jsp 

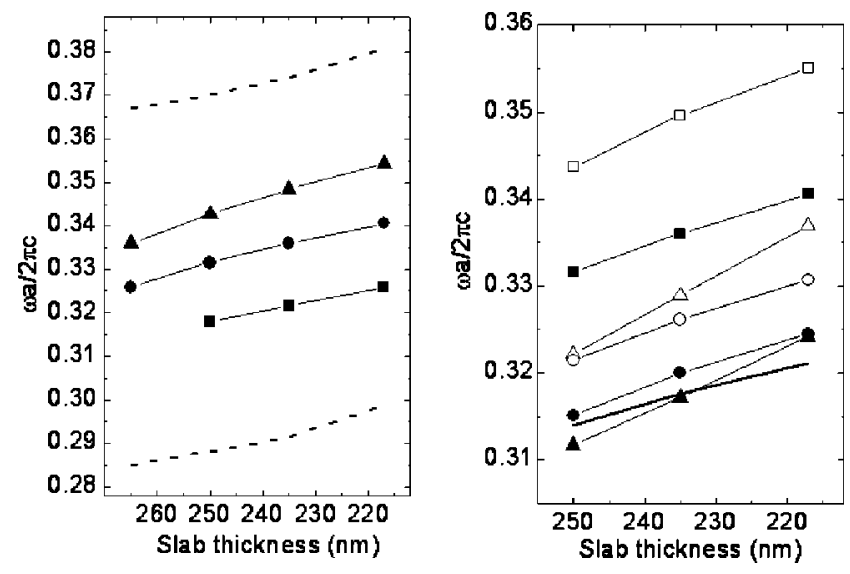

FIG. 3. Left panel: change in the normalized frequencies of emission for different modes of the H5 cavity of Fig. 1 versus the thickness of the slab. The calculated position of the border of the valence band and conduction band of the photonic band gap is shown in dashed lines. Right panel: variation in the normalized frequency of the emission corresponding to selected cavity modes of H1 (triangles), H3 (circles) and H5 (squares) cavities versus the thickness of the slab. The solid line shows the evolution trend of the highest intensity mode of an H1 cavity obtained by 3D FDTD calculations.

variation is around $2 \mathrm{~nm}$ per etched nanometer. We have included the variation of the top of the valence band and the bottom of the conduction band with the thickness for comparison. The rate at which the modes shift towards higher energies as the thickness decreases is similar to the calculated variation of the borders of the band gap.

Figure 3 shows the variation of the normalized frequency of the peaks corresponding to selected cavity modes versus the thickness for $\mathrm{H} 1, \mathrm{H} 3$ and $\mathrm{H} 5$ cavities. In the case of $\mathrm{H} 3$ and $\mathrm{H} 5$ cavities only two of the peaks from all of the cavity modes observed are shown for clarity. The shift of the modes in the $\mathrm{H} 3$ and $\mathrm{H} 5$ cavities changes with the slab thickness at the same rate, whereas the change in a $\mathrm{H} 1$ cavity is faster. In order to analyze the shift of the cavity modes we considered that the $\mathrm{H} 1$ cavity has a reduced number of modes, which can be easily identified through different computing simulations. We have performed three-dimensional finite-difference time-domain (3D-FDTD) calculations ${ }^{20}$ of a H1 cavity in a slab with variable thickness to obtain the normalized frequencies of the cavity modes. Tapered holes with sides of $5^{\circ}$ inclination have been also used. The shift rate obtained for the mode with higher intensity is shown in Fig. 3. Additional 3D-FDTD calculations show that the shift in the peaks is affected by the shape of the holes. We have also obtained that as the slope of the sides increases, the shift rate of the modes decreases. However, this factor alone produces a variation in frequencies that is much less (around five times smaller for a change from 0 to $5^{\circ}$ ) than what is experimentally measured. As the photonic band gap changes (in size and in position) with the thickness, the cavity modes also change in the same direction (toward higher energies). We would expect a very similar behavior for the case of modal emission in a photonic crystal laser. Nevertheless, the lasing mode may change depending on the position of the excitation spot and other experimental conditions which makes it much more difficult to identify the frequency of the lasing mode than in the case of the spontaneous emission study.

In summary, we have shown how the variation in the thickness of a photonic crystal microcavity on a slab significantly alters the emission wavelengths of the cavity modes. The shift in normalized frequencies follows the shift of the photonic band gap of the triangular lattice calculated by three-dimensional PWE methods. This effect shows the important effects of the third dimension in planar photonic crystal structures and it can be very useful for fine laser tuning after device fabrication. Further work is necessary to assess how the lasing modes may shift in a microcavity depending on the thickness of the slab.

A. R. Alija thanks an FPU fellowship AP2002-0474 and L. J. Martínez an I3P fellowship. P. A. Postigo and A. GarcíaMartín would like to thank "Ramón y Cajal" contracts and projects CAM 07N/0059/02, CICYT TIC2002-C03-03, DPI2001-0024-C03-01.

${ }^{1}$ O. Painter, R. K. Lee, A. Scherer, A. Yariv, J. D. O’Brien, P. D. Dapkus, and I. Kim, Science 284, 1819 (1999).

${ }^{2}$ H. Benisty, S. Olivier, C. Weisbuch, M. Agio, M. Kafesaki, C. M. Soukoulis, M. Qiu, M. Swillo, A. Karlsson, B. Jaskorzynska, A. Talneau, J. Moosburger, M. Kamp, A. Forchel, R. Ferrini, R. Houdré, and U. Oesterle, IEEE J. Quantum Electron. 38, (2002).

${ }^{3}$ C. Monat, C. Seassal, X. Letartre, P. Regreny, M. Gendry, P. Rojo Romeo, P. Viktorovitch, M. Le Vassor d'Yerville, D. Cassagne, J. P. Albert, E. Jalaguier, S. Pocas, and B. Aspar, J. Appl. Phys. 93(1), 23-21 (2003).

${ }^{4}$ H.-Y. Ryu, S.-H. Kwon, Y.-J. Lee, Y.-H. Lee, and J.-S. Kim, Appl. Phys. Lett. 80(19), 2476-3478 (2002).

${ }^{5}$ C. Reese, C. Becher, A. Imamoglu, E. Hu, B. D. Gerardot, P. M. Petroff, Appl. Phys. Lett. 78(16), 2279-2281 (2001).

${ }^{6}$ P. Pottier, C. Seassal, X. Letartre, J. L. Leclercq, P. Viktorovitch, D. Cassagne, and C. Jouanin, J. Lightwave, J. Lightwave Technol. 17, 2058 (1999).

${ }^{7}$ K. Hennessy, C. Reese, A. Badolato, C. F. Wang, A. Imamoglu, P. M. Petroff, E. Hua, G. Jin, S. Shi, and D. W. Prather, Appl. Phys. Lett. 83(18), 3650-3652 (2003).

${ }^{8}$ C. Monat, C. Seassal, X. Letartre, P. Regreny, P. Rojo-Romeo, P. Viktorovitch, M. L. d'Yerville, D. Cassagne, J. P. Albert, E. Jalaguier, S. Pocas, and B. Aspar, IEEE J. Quantum Electron. 39(3), 419-425 (2003).

${ }^{9}$ N. Yokouchi, A. J. Danner, and K. D. Choquette, Appl. Phys. Lett. 82(9), 1344-1346 (2003).

${ }^{10}$ Y. Tanaka, T. Asano, Y. Akahane, B.-S. Song, and S. Noda, Appl. Phys. Lett. 82, 1661 (2003).

${ }^{11}$ L. C. Andreani and M. Agio, IEEE J. Quantum Electron. 38, 891 (2002).

${ }^{12}$ B. Wild, R. Ferrini, R. Houdre, M. Mulot, S. Anand, and C. J. M. Smith, Appl. Phys. Lett. 84(6), 846-848 (2004).

${ }^{13}$ M. Loncar and A. Scherer, Appl. Phys. Lett. 82(26), 4648-4650 (2003).

${ }^{14}$ P. A. Postigo, D. Golmayo, H. Gómez, D. Rodríguez, and M. L. Dotor, Jpn. J. Appl. Phys., Part 2 41, L565 (2002).

${ }^{15}$ J. R. Sendra, G. Armelles, and J. Anguita, Semicond. Sci. Technol. 11, 238 (1996).

${ }^{16}$ M. Boroditsky, R. Vrijen, T. F. Krauss, R. Coccioli, R. Bhat, and E. Yablonovitch, J. Lightwave Technol. 17, 11 (1999).

${ }^{17}$ C. J. M. Smith, R. M. De La Rue, M. Rattier, S. Olivier, H. Benisty, C. Weisbuch, T. F. Krauss, R. Houdré, and U. Oesterle, Appl. Phys. Lett. 78, 1487 (2001).

${ }^{18}$ T. Baba, N. Kamizawa, and M. Ikeda, Physica B 227, 415 (1996).

${ }^{19}$ J. R. Sendra and J. Anguita, Jpn. J. Appl. Phys., Part 2 33, L390 (1994).

${ }^{20}$ Available at http://alioth.debian.org/projects/tessa 